\title{
Early Treatment of COVID-19 Disease: A Missed Opportunity
}

\author{
Jamie I. Forrest (1) · Craig R. Rayner · Jay J. H. Park $\cdot$ Edward J. Mills
}

Received: August 22, 2020 / Accepted: September 23, 2020 / Published online: October 13, 2020

(C) The Author(s) 2020, corrected publication 2020

\begin{abstract}
Antivirals have demonstrated efficacy in treating other infectious diseases in early stages of disease, reducing morbidity, mortality, and the likelihood of onward transmission. At the time of writing, more than 1900 clinical trials are registered globally to assess the efficacy and safety of candidate therapeutics for COVID-19. The majority of these trials are designed to evaluate the comparative efficacy and safety of
\end{abstract}

J. I. Forrest · J. J. H. Park · E. J. Mills (ه)

Cytel Canada Health Inc., Vancouver, BC, Canada

e-mail: edward.mills@cytel.com

E. J. Mills

Faculty of Health Sciences, McMaster University,

Hamilton, ON, Canada

C. R. Rayner

Certara, NJ, USA

J. I. Forrest · J. J. H. Park

Faculty of Medicine, University of British Columbia,

Vancouver, BC, Canada

C. R. Rayner

Monash Institute of Pharmaceutical Sciences,

Monash University, Melbourne, VIC, Australia candidate therapeutics for the treatment of COVID-19 to prevent death among populations of hospitalized patients with advanced disease. Yet, emerging epidemiological evidence now indicates that the majority of those infected with the SARS-CoV-2, while still infectious, experience minimal or mild disease symptomology. Like HIV and hepatitis C that pioneered treatment as prevention, there is a missed opportunity for trials of early pharmaceutical intervention for COVID-19 disease evaluating not only reductions in morbidity and mortality but also transmissibility. We discuss this clinical research gap within an historical context of viral treatment as prevention for HIV and hepatitis C, and comment on the challenges and opportunities for clinical research of candidate therapeutics for early COVID-19 disease.

Keywords: Clinical trials; COVID-19; Early treatment; Treatment as prevention 


\section{Key Summary Points}

The majority of clinical trials evaluating candidate therapeutic interventions for the treatment of COVID-19 disease are among populations of hospitalized and severely sick patients

Antivirals, shown to be effective in early treatment of other infectious diseases, should continue to be explored in trials among patients with early COVID-19 disease

Lessons from other diseases like HIV and hepatitis $\mathrm{C}$ demonstrate that early treatment, coupled with frequent and accessible testing, can reduce morbidity, mortality, and likelihood of transmission

Investigations of candidate therapeutics for early COVID-19 disease should not compete with ongoing vaccine trials, and given the challenges for distribution and financing of a successful vaccine, early treatment could be important to mitigating the pandemic

\section{DIGITAL FEATURES}

This article is published with digital features to facilitate understanding of the article. To view digital features for this article go to https://doi. org/10.6084/m9.figshare.12967049.

With more than 28 million people reportedly infected with SARS-CoV-2 globally, resulting in more than 900,000 deaths [1], the COVID-19 global pandemic has ushered a new era of rapid and cooperative global health research. At the time of writing there are over $1900(n=1970)$ registered clinical trials globally evaluating candidate therapeutics for the treatment of COVID-19 disease (https://www. covid19-trials.com) [2]. The majority $(n=1129 ; 57 \%)$ of these trials aim to report the

comparative efficacy and safety of candidate therapeutics on reducing death among populations of hospitalized and severely sick patients. These trials, such as the RECOVERY trial, have since generated important findings to support the use therapeutics such as the corticosteroid dexamethasone, shown to significantly reduce mortality among specific populations of hospitalized patients with advanced COVID-19 disease [3].

While these trials are important for the development of effective therapeutics to prevent death among hospitalized patients with advanced COVID-19 disease, emerging epidemiological evidence indicates that a majority those infected with the SARS-CoV-2 either do not develop any symptoms of COVID-19 disease or are able to manage disease symptoms without being admitted to hospital [4]. However, despite a majority of those infected either with no symptoms or mild symptoms, measurements of viral load between symptomatic and asymptomatic patients are reportedly similar [5], indicating that all infected populations can transmit the virus.

With trials among hospitalized patients with COVID-19, we identified only 119 (6.0\%) registered trials reportedly evaluating the efficacy of candidate interventions to reduce hospitalization or transmission among ambulatory populations with an early diagnosis of SARSCoV-2 infection, or as a prophylactic therapeutic for highly exposed populations (https:// www.covid19-trials.com). The limited number of trials investigating therapeutics for early treatment of COVID-19 disease is disappointing since early treatment will likely yield the greatest treatment benefits to both patients and communities.

Evidence to support prioritizing clinical trials for COVID-19 among early infected populations can be drawn from historical clinical research informing the treatment of HIV and hepatitis C. The evolution of clinical research to treat and prevent both these infectious diseases has led to scientific and clinical consensus that early diagnosis and initiation of therapy is shown not only to reduce morbidity and mortality but also significantly reduce the likelihood of viral transmission [6]. 


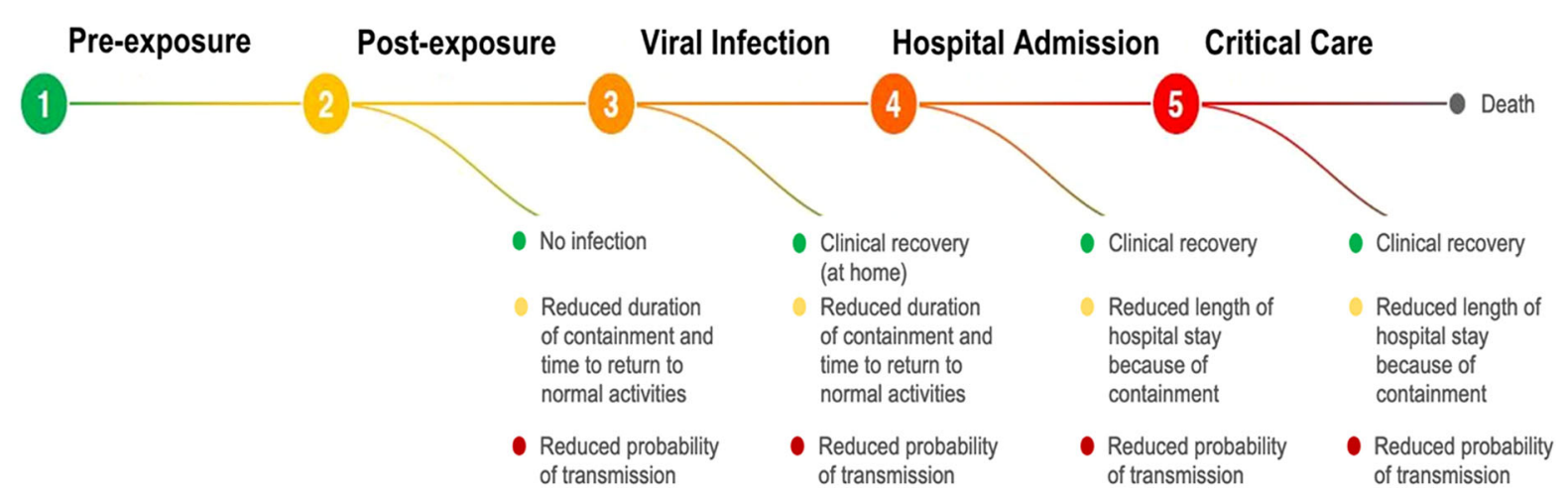

Fig. 1 Therapeutic targets and populations of interest to clinical intervention of COVID-19

COVID-19 clinical trials target at least five stages of the disease process: (1) pre-exposure prophylaxis (PrEP), (2) post-exposure prophylaxis (PEP), (3) outpatient treatment, (4) hospitalized patients, and (5) late-stage critical care (admission to intensive care unit, ICU) (Fig. 1). Although we are only beginning to fully understand disease progression of COVID-19, it is generally understood that the initial period of infection is driven primarily by viral replication, often targeting lung cells, that results in a high local virus titre before the body is able to mount an adequate immune response [7]. For this reason, there has been enthusiasm for the use of antivirals as early treatment as well as for prophylaxis. However, to date, no clinical trials have demonstrated the efficacy of any interventions in these patient settings. This should not lead to pessimism. Drawing from HIV and other diseases, monotherapies rarely exhibit optimal treatment effects and combination strategies should be considered in clinical trials [8].

The priority for early treatment is not limited to individual patient recovery. The downstream effects of preventing disease progression represent some of the most immediate public health challenges of this pandemic. Preventing individuals from worsening COVID disease reduces their likelihood of hospital admission, thereby reducing medical resource costs; reducing utilization and exposure to healthcare workers; and reducing transmission to others. PrEP and PEP are among the many interventions being evaluated for COVID-19. PrEP represents the earliest possible treatment opportunity, even before exposure, but utilizes the therapeutic effects of early treatment drugs. For example, the COPCOV trial (NCT04303507) is enrolling 40,000 healthcare workers in Thailand and the UK to determine if treatments such as hydroxychloroquine reduce the likelihood of acquiring COVID-19. PEP also represents early treatment based on empiric assumptions of infection. Utilizing treatment as prevention as a public health strategy borrows strongly from HIV, hepatitis $\mathrm{C}$, and tuberculosis prevention campaigns [9] For COVID-19, early treatment that reduces viral shedding should be considered a priority outcome, as decreased transmission to others has both individual and community benefits. Although the amount of exposure to virus required for successful transmission is unknown, treated individuals may be less likely to transmit compared to untreated infected persons. Early treatment is very likely to be costeffective. By reducing the period of infectiousness, there are important opportunities to shorten the duration of containment and quarantine, and realise both quality of life and economic benefits via reduced direct and indirect costs associated with isolation infection control practices.

By reducing the probability of clinical deterioration and hospital admission, there is also the potential for significant cost avoidance. Considering the average cost of hospitalization due to COVID-19 in the USA is US\$73,000 per patient, a drug costing less than US\$3000 (e.g. the approximate cost of remdesivir) is likely cost-effective when considering likely reduced transmission and their subsequent probability 
of hospitalization. Repurposed drugs would likely be much cheaper than novel ones, but number needed to treat (NNT) to prevent hospitalization should be estimated for early interventions. Given the incidence of COVID in lowand middle-income countries, there is a priority for low-cost interventions.

Antivirals are a cornerstone of pandemic preparedness and pandemic response. They can be directed for treatment of infection and in situations where vaccines are ineffective or unavailable, and they are proven to treat and prevent the transmission of infectious diseases. At the patient level, viral infection and replication results in a host response and disease manifestations, and therapeutics that target this disease progression, particularly those in early disease stages, can minimize the inflammatory host response that leads to poor patient clinical outcomes [10]. By reducing the high initial viral titres and duration of viral shedding of an individual, antivirals can importantly also reduce the period of infectiousness, resulting in a decreased length of home isolation or length of stay in hospital. For example, oseltamivir, approved by the US Food and Drug Administration (FDA) for both treatment and prophylaxis of influenza, has been shown to decrease transmission among households and prevent community outbreaks [7].

Like therapeutics for HIV and hepatitis C $[11,12]$, antivirals have been utilised in public health contexts to reduce community transmission [13]. Interdisciplinary modelling studies linking antiviral pharmacology impact on individual viral shedding to community spread to health economics [14] can be used to inform optimal positioning of antiviral treatment interventions within the patient disease journey and health care system for maximum "individual" and/or "population health" benefit. In contrast to the ICU or inpatient environments, it stands to reason that early infections identified in an outpatient setting have the highest potential for public health impact. In this context, cessation of viral shedding is an endpoint that has as high importance as an indicator of patient clinical improvement. The evaluation of combination antivirals is likely necessary to identify therapeutics that achieve both these clinical and virologic benefits [15].

While this theoretical scientific support for antiviral therapeutics for early COVID-19 disease is promising, there are substantial challenges posed to clinical trialists aiming to evaluate candidate interventions. First, while the epidemiology of COVID-19 has been extensively reviewed [16], defining and recruiting patient populations with early COVID-19 disease is a challenge for trialists. For the limited number of ongoing trials evaluating candidate therapeutics among patient populations with early COVID-19 disease, differences in study inclusion criteria may limit the comparability of study findings. For example, the inclusion criteria for participation in the TOGETHER Trial (https://www.togethertrial.com) with recruitment sites in Washington State, Brazil, and South Africa define its study population of patients with early COVID-19 infection by those who present to a clinic with COVID-19 symptoms with a confirmed RT-PCR diagnosis and self-report the onset of these symptoms within $72 \mathrm{~h}$ prior to diagnosis. These study inclusion criteria differ from those of a trial in France, for example, that reported findings on the comparative efficacy of therapeutics, in an early infected patient population recruited through a mass testing campaign and included both patients with a confirmed diagnosis who were both symptomatic and asymptomatic, and their contacts [17].

Another major challenge for trialists conducting studies evaluating the comparative efficacy of interventions among early COVID-19 disease populations is identifying and measuring the right outcome. Trials among this population are most often powered to detect differences in a primary outcome of hospitalization. While this outcome is important for identifying effective therapeutics that slow disease progression and the need for inpatient care, secondary outcome measures are still needed to identify effective therapeutics that reduce viral load and thus the likelihood of transmission. Clinical trials evaluating both repurposed and novel therapeutic agents in this population with different mechanisms of action 
should consider outcomes that measure both disease progression and changes in viral load.

Adding to these challenges is that trials among early COVID-19 disease populations are more expensive to conduct than trials in outpatient settings. Early disease populations are more resource-intensive to identify and retain in a study participation, requiring home visits by study staff and follow-up by telemedicine with each patient. For trials in this population to succeed, research funders must acknowledge the important differences between hospitalized and early disease outpatient populations, and commit to appropriate funding streams to support these trials.

The search for effective therapeutics among early COVID-19 disease populations must also be scalable and acceptable among a general population. Trials of lopinavir-ritonavir, for example, have reported a high rate of discontinuation due to adverse events [18]. Researchers must also consider the implications of overtreating COVID-19. The vast majority of those infected with COVID-19 recover without any medical intervention, so considerations for appropriate use of early treatment must still be investigated. Finally, a research agenda with the aim of identifying effective therapeutics for the treatment of early COVID-19 disease should not be considered a should not compete with a parallel research agenda for vaccine development. While a number of vaccine candidates are demonstrating promise, little is known about how long these vaccines will provide protection, and even if a strong candidate emerges, global scale-up and equitable access will present important challenges.

While early treatment for infectious diseases to prevent morbidity and mortality and reduce community transmission is not a new idea, there is limited attention to this approach among clinical trialists evaluating therapeutics for COVID-19 in this population. Adapting this strategy of expanding access to early treatment for COVID-19 poses many challenges, and necessitates a unified global research agenda that recognizes the promise of early treatment for COVID-19 disease and prioritizes research funding for multi-site clinical trials of this population. In the absence of an effective and widely available vaccine, failing to prioritize clinical research among early infected populations may be a missed opportunity.

\section{ACKNOWLEDGEMENTS}

Funding. The authors acknowledge the funding support of the Bill and Melinda Gates Foundation and the Thistledown Foundation for this commentary and the journal's Rapid Service Fee.

Authorship. All named authors meet the International Committee of Medical Journal Editors (ICMJE) criteria for authorship for this article, take responsibility for the integrity of the work as a whole, and have given their approval for this version to be published.

Disclosures. Jamie I. Forrest, Craig R. Rayner, Jay J. H. Park and Edward J. Mills have nothing to disclose.

Compliance with Ethics Guidelines. This article is based on previously conducted studies and does not contain any studies with human participants or animals performed by the authors.

Open Access. This article is licensed under a Creative Commons Attribution-NonCommercial 4.0 International License, which permits any non-commercial use, sharing, adaptation, distribution and reproduction in any medium or format, as long as you give appropriate credit to the original author(s) and the source, provide a link to the Creative Commons licence, and indicate if changes were made. The images or other third party material in this article are included in the article's Creative Commons licence, unless indicated otherwise in a credit line to the material. If material is not included in the article's Creative Commons licence and your intended use is not permitted by statutory regulation or exceeds the permitted use, you will need to obtain permission directly from the copyright holder. To view a copy of this licence, 
visit http://creativecommons.org/licenses/bync/4.0/.

\section{REFERENCES}

1. Johns University \& Medicine. Coronavirus Resource Center. 2020. https://coronavirus.jhu.edu. Accessed 10 Sept 2020

2. Thorlund K, Dron L, Park J, Hsu G, Forrest JI, Mills EJ. A real-time dashboard of clinical trials for COVID-19. Lancet Digit Health. 2020;2(6): e286-e287287.

3. Horby P, Lim WS, Emberson JR, et al. Dexamethasone in hospitalized patients with Covid-19-preliminary report. N Engl J Med. 2020. https://doi. org/10.1056/NEJMoa2021436.

4. Gao Z, Xu Y, Sun C, et al. A systematic review of asymptomatic infections with COVID-19. J Microbiol Immunol Infect. 2020. https://doi.org/10. 1016/j.jmii.2020.05.001.

5. Huff HV, Singh A. Asymptomatic transmission during the COVID-19 pandemic and implications for public health strategies. Clin Infect Dis. 2020. https://doi.org/10.1093/cid/ciaa654.

6. Group ISS. Initiation of antiretroviral therapy in early asymptomatic HIV infection. N Engl J Med. 2015;373(9):795-807.

7. Abena PM, Decloedt EH, Bottieau E, et al. Chloroquine and hydroxychloroquine for the prevention or treatment of COVID-19 in Africa: caution for inappropriate off-label use in healthcare settings. Am J Trop Med Hyg. 2020;102(6):1184-8.

8. Bartlett JA, Fath MJ, Demasi R, et al. An updated systematic overview of triple combination therapy in antiretroviral-naive HIV-infected adults. AIDS. 2006;20(16):2051-64.

9. Montaner JS, Hogg R, Wood E, et al. The case for expanding access to highly active antiretroviral therapy to curb the growth of the HIV epidemic. Lancet. 2006;368(9534):531-6.

10. García LF. Immune response, inflammation, and the clinical spectrum of COVID-19. Front Immunol. 2020;11:1441.

11. Hagan LM, Wolpe PR, Schinazi RF. Treatment as prevention and cure towards global eradication of hepatitis C virus. Trends Microbiol. 2013;21(12): 625-33.

12. Hull MW, Montaner JS. HIV treatment as prevention: the key to an AIDS-free generation. J Food Drug Anal. 2013;21(4):S95-s101.

13. Bershteyn A, Klein DJ, Eckhoff PA. Age-targeted HIV treatment and primary prevention as a 'ring fence' to efficiently interrupt the age patterns of transmission in generalized epidemic settings in South Africa. Int Health. 2016;8(4):277-85.

14. Kamal MA, Smith PF, Chaiyakunapruk N, et al. Interdisciplinary pharmacometrics linking oseltamivir pharmacology, influenza epidemiology and health economics to inform antiviral use in pandemics. Br J Clin Pharmacol. 2017;83(7):1580-94.

15. Dodds MG, Krishna R, Goncalves A, Rayner CR. Model-informed drug repurposing: viral kinetic modelling to prioritize rational drug combinations for COVID-19. Br J Clin Pharmacol. 2020. https:// doi.org/10.1111/bcp.14486.

16. Gabutti G, d'Anchera E, Sandri F, Savio M, Stefanati A. Coronavirus: update related to the current outbreak of COVID-19. Infect Dis Ther. 2020;9(2):1-13.

17. Million M, Lagier JC, Gautret P, et al. Early treatment of COVID-19 patients with hydroxychloroquine and azithromycin: a retrospective analysis of 1061 cases in Marseille, France. Travel Med Infect Dis. 2020;35:101738.

18. Cao B, Wang Y, Wen D, et al. A trial of lopinavirritonavir in adults hospitalized with severe Covid19. N Engl J Med. 2020;382(19):1787-99. 